\title{
Pharmacokinetic and Pharmacodynamic Analysis of Hyperthermic Intraperitoneal Oxaliplatin-Induced Neutropenia in Subjects with Peritoneal Carcinomatosis
}

\author{
Belén Valenzuela, ${ }^{1,4}$ Ricardo Nalda-Molina, ${ }^{2}$ Pere Bretcha-Boix, ${ }^{1}$ Vanesa Escudero-Ortíz, ${ }^{1}$ Maria José Duart, ${ }^{2}$ \\ Vicente Carbonell, ${ }^{3}$ Manuel Sureda, ${ }^{1}$ José Pascual Rebollo, ${ }^{1}$ Josep Farré, ${ }^{1}$ Antonio Brugarolas, ${ }^{1}$ \\ and Juan José Pérez-Ruixo ${ }^{2}$
}

\begin{abstract}
Received 4 October 2010; accepted 14 December 2010; published online 6 January 2011
Abstract. The objective of this study was to characterize the pharmacokinetics and the time course of the neutropenia-induced by hyperthermic intraperitoneal oxaliplatin (HIO) after cytoreductive surgery in cancer patients with peritoneal carcinomatosis. Data from 30 patients who received $360 \mathrm{mg} / \mathrm{m}^{2}$ of HIO following cytoreductive surgery were used for pharmacokinetic-pharmacodynamic (PK/PD) analysis. The oxaliplatin plasma concentrations were characterized by an open two-compartment pharmacokinetic model after first-order absorption from peritoneum to plasma. An oxaliplatin-sensitive progenitor cell compartment was used to describe the absolute neutrophil counts in blood. The reduction of the proliferation rate of the progenitor cells was modeled by a linear function of the oxaliplatin plasma concentrations. The typical values of oxaliplatin absorption and terminal half-lives were estimated to be 2.2 and $40 \mathrm{~h}$, with moderate interindividual variability. Oxaliplatin reduced the proliferation rate of the progenitor cells by $18.2 \%$ per $\mathrm{mg} / \mathrm{L}$. No patient's covariates were related to oxaliplatin PK/PD parameters. Bootstrap and visual predictive check evidenced the model was deemed appropriate to describe oxaliplatin pharmacokinetics and the incidence and severity of neutropenia. A peritoneum oxaliplatin exposure of 65 and $120 \mathrm{mg} \cdot \mathrm{L} / \mathrm{h}$ was associated with a $20 \%$ and $33 \%$ incidence of neutropenia grade 4. The time course of neutropenia following HIO administration was well described by the semiphysiological PK/PD model. The maximum tolerated peritoneum oxaliplatin exposure is $120 \mathrm{mg} \mathrm{L} / \mathrm{h}$ and higher exposures should be avoided in future studies. We suggest the prophylactic use of granulocyte colony stimulating factor for patients treated with HIO exposure higher than $65 \mathrm{mg} \mathrm{L} / \mathrm{h}$.
\end{abstract}

KEY WORDS: hyperthermic intraperitoneal chemotherapy (HIPEC); NONMEM; oxaliplatin; peritoneal carcinomatosis; pharmacodynamics; pharmacokinetics.

\section{INTRODUCTION}

For many patients with peritoneal carcinomatosis (PC) secondary to intra-abdominal cancers, tumor progression in the peritoneum is the sole life-limiting component of disease and one of the most common causes of cancer incurability. However, there are no PC treatment approved by regulatory agencies, and the development of new therapies to manage this life-threatening condition could fulfill an unmet medical need. In this context, several Phase I/II clinical studies in gastric cancer (1-3), mesothelioma (4), colorectal (5), or ovarian carcinoma (6) have shown promising results in treating macroscopic PC with cytoreductive surgery and residual PC with hyperthermic intraperitoneal chemotherapy

\footnotetext{
${ }^{1}$ Platform of Oncology, USP Hospital San Jaime, Partida de la Loma s/n, 03184 Torrevieja, Alicante, Spain.

${ }^{2}$ Pharmaceutical Sciences Department, University Miguel Hernandez, Alicante, Spain.

${ }^{3}$ AINIA Technology Centre, Paterna, Valencia, Spain.

${ }^{4}$ To whom correspondence should be addressed. (e-mail: belen. valenzuela@usphospitales.com)
}

(HIPEC) (7). The rationale for this treatment is based on experimental studies showing that drug penetration is limited to a few cell layers under the surface of the tumor (8), and consequently, intraperitoneal chemotherapy must be immediately administered after the cytoreductive surgery in order to achieve the maximal cytotoxic activity on residual tumor cells before they get trapped in the postoperative fibrin adhesions (9). In addition, the intratumoral cytotoxic activity can be enhanced by administering highly permeable drugs at relatively high doses with hyperthermia. The efficacy of this approach has been evidenced in Phase III studies in colorectal cancer patients $(10,11)$.

The ideal drug for HIPEC should penetrate into the tumor, have a low diffusion into the subperitoneal space and capillary endothelial in order to avoid excessive drug systemic exposure, and have a temperature dependent cytotoxic activity. Therefore, drugs rapidly metabolized and/or excreted from the body should be preferred over others as they should decrease the systemic exposure and the risk of toxicity. Consequently, large hydrophilic compounds with limited permeability across an intact peritoneal membrane have been preferred to small lipophilic compounds (12). In this context, 
cisplatin (13), mytomicin C (14), carboplatin (15), paclitaxel (16), irinotecan (17), and oxaliplatin (18) have been used as chemotherapy agents in HIPEC because their cytotoxic activity is enhanced with hyperthermia. Although comparative studies across these drugs have not been performed to date, the results obtained with oxaliplatin are encouraging. In a retrospective analysis in patients with resectable PC, Elias et al. have shown that surgical cytoreduction followed by HIPEC with oxaliplatin prolongs median survival from 23.9 to 62.7 months and increase the 5-year survival rate from $13 \%$ to $51 \%$ with respect to standard palliative surgery and chemotherapy (19).

Oxaliplatin, a diaminocyclohexane-platinum compound active in a variety of solid tumor types, licensed in the USA and Europe for the treatment of colorectal cancer, is an attractive agent for HIPEC because its cytotoxicity is significantly increased by hyperthermia $(20,21)$ and the intratumoral penetration is optimal (22). However, systemic exposure to oxaliplatin increases the risk of hematological toxicity and peripheral sensory neuropathy, which have been described as dose-limiting toxicities after intravenous treatment (23). Therefore, it is important to achieve the maximum oxaliplatin exposure in the peritoneal cavity with minimum access to systemic circulation, in order to balance the cytotoxic activity and the risk of toxicity. Several Phase I dose-escalation studies in subjects with PC were conducted to determine the maximum tolerated dose of hyperthermic intraperitoneal oxaliplatin (HIO) and characterized its pharmacokinetics (PK) as a single agent (24-26). In these studies, intraperitoneal doses ranging from 200 to $460 \mathrm{mg} / \mathrm{m}^{2}$ were administered during 0.5 to $2 \mathrm{~h}$ and oxaliplatin exhibited linear and time-independent PK in both plasma and peritoneum. While oxaliplatin peritoneal concentrations decline in an exponential manner with a halflife of 30 to $40 \mathrm{~min}(22,27,28)$, oxaliplatin plasma concentrations increase to reach the maximum shortly after the end of the intraperitoneal infusion. The mean value of apparent oxaliplatin absorption rate constant $\left(k_{\mathrm{a}}\right)$ from peritoneum to plasma was close to $1.4 \mathrm{~h}^{-1}(22,27,28)$. After treatment with $\mathrm{HIO}$, oxaliplatin plasma concentrations declined in a bi-exponential manner resembling to the PK profiles observed after an intravenous administration. At an oxaliplatin dose level of $460 \mathrm{mg} / \mathrm{m}^{2}$, the peak concentration $\left(C_{\max }\right)$ was estimated to be 25 -fold higher in peritoneum $(330 \mu \mathrm{g} / \mathrm{mL})$ than in plasma $(13.2 \mu \mathrm{g} / \mathrm{mL})(24,25)$ which indicates an increased oxaliplatin tumor exposure and, potentially, a more efficacious treatment for residual PC than its intravenous administration. However, the pharmacokinetic parameters obtained from plasma concentrations achieved following HIO vary substantially from one study to others reflecting differences in relation to (1) the analyte (ultrafiltrate $v s$ total platinum), (2) analytical method for measuring plasma platinum content (atomic absorption spectroscopy, liquid chromatography, or inductively coupled plasma atomic emission spectrometry), and (3) the carrier solution used (isotonic, hypotonic, or hypertonic solutions) (29). In this context, is not surprising that the estimated oxaliplatin plasma clearance ranged from $6.68 \mathrm{~L} / \mathrm{h} / \mathrm{m}^{2}(27)$ to $28.4 \mathrm{~L} / \mathrm{h} / \mathrm{m}^{2}(28)$, while the volume of distribution is estimated to be $15 \mathrm{~L}(27)$.

In these studies, the most frequent and severe adverse events related with oxaliplatin were neutropenia and thrombocytopenia (24-27). Stewart et al. (26) reported that hematologic toxicity grade was related to the extent and the rate of oxaliplatin systemic absorption at $250 \mathrm{mg} / \mathrm{m}^{2}$ administered during $2 \mathrm{~h}$. However, to date, there are not quantitative longitudinal analyses exploring the effect of oxaliplatin pharmacokinetics on the time course of hematological toxicity after its intraperitoneal administration. In this study, we characterize the oxaliplatin pharmacokinetics in peritoneum and plasma when is administered in an icodextrin $4 \%$ carrier solution and establish its relationship with the time course of absolute neutrophil counts (ANC) in patients with $\mathrm{PC}$ receiving $\mathrm{HIO}$ after cytoreductive surgery. A semi-mechanistic population pharmacokinetic and pharmacodynamic (PK/PD) model previously developed (30) was used to analyze the data and the effect of patient demographics and/or physiopathological factors on oxaliplatin PK/PD parameters. Finally, the relationship between oxaliplatin concentrations in peritoneum, the duration of the HIO and the incidence of severe neutropenia was explored in order to establish the maximum tolerated oxaliplatin exposure, which will be critical to optimize the design of future clinical studies with oxaliplatin in this setting.

\section{MATERIALS AND METHODS}

Study Design and Subject Eligibility Criteria. Data from 30 subjects included in a single-arm study investigating the safety, tolerability, pharmacokinetics, and pharmacodynamics of HIO after cytoreductive surgery were analyzed. Adult patients were eligible if they had confirmation of PC without extra-abdominal metastasis. Other eligibility criteria included a World Health Organization performance status of 0 to 2, anticipated life expectancy of at least 3 months. Previous anticancer radiation therapy and/or chemotherapy, if given, had to be discontinued for at least 4 weeks before entry into the study, or 6 weeks in the case of pretreatment with nitrosoureas or mitomycin C. Patients were required to have a negative pregnancy test (only for female patients with reproductive potential), and normal hepatic and renal function, defined as bilirubin $\leq 1.5$ times the upper limit of normality, aspartate aminotransferase (AST) and alanine aminotransferase $($ ALT $) \leq 2.5$ times the upper limit of normality, and serum creatinine $\leq 1.5$ times the upper limit of normality. An acceptable bone marrow function, defined as white blood cells $>3.5 \times$ $10^{9} / \mathrm{L}$, neutrophil count $>1.5 \times 10^{9} / \mathrm{L}$, and platelets $>100.0 \times 10^{9} / \mathrm{L}$ was needed. Patients with one or more of the following criteria were not selected: active infection; central nervous system metastases; peripheral neuropathy >grade 2; allogenic transplant; prior extensive radiation therapy $(>25 \%$ of bone marrow reserve); prior bone marrow transplantation or high dose chemotherapy with bone marrow or stem cell rescue; concurrent radiation therapy, chemotherapy, hormonal therapy, or immunotherapy; participation in a clinical trial involving an investigational drug in the past 30 days or concurrent enrollment in another investigational trial; and any coexisting medical condition that was likely to interfere with study procedures and/or results.

The study was conducted at the USP Hospital San Jaime (Torrevieja, Spain) between 2006 and 2009 in accordance with principles for human experimentation as defined in the 
International Conference on Harmonization for Good Clinical Practice guidelines and the principles of the Declaration of Helsinki. The study was approved by the corresponding Investigational Review Board and informed consent was obtained from each subject after being advised of the potential risks and benefits, as well as, the investigational nature of the study (31). The primary tumor type of the eligible patients were ovarian $(n=10)$, colorectal $(n=9)$, appendiceal $(n=5)$, gastric $(n=3)$, endometrial $(n=2)$, and primary papilar $(n=1)$. A summary of patient characteristics at baseline is presented in Table I.

Surgical Procedure. A xiphopubic midline laparotomy was carried out to examine the tumor load in the abdominal cavity. To obtain the peritoneal cancer index (32), the abdomen was divided into 13 areas numbered from 0 to 12 , as described elsewhere (33), and cytological samples and biopsies were taken from each area. Resection of the primary tumor when present was carried out according to regional lymphadenectomy with correct margins. In carcinomatosis with the primary tumor in situ and in metachronous cases, peritonectomies and debulking were carried out as required and extensive systematic peritonectomies were not performed. The mesenteric peritoneum was not extensively removed, and acceptable small bowel resections were guided by maximal tumor volume locations. Remaining malignant granulations were destroyed using electrosurgical fulguration. This aggressive surgical cytoreduction was performed with the aim to reach complete resection or, if not possible, to

Table I. Summary of Patient Characteristics at Baseline

\begin{tabular}{|c|c|c|}
\hline Subject characteristics $(N=30)$ & Mean (SD) & Range \\
\hline Age (year) & $57.9(10.5)$ & $32.0-75.0$ \\
\hline Body Weight (kg) & $69.3(12.1)$ & $42.0-90.0$ \\
\hline Body Surface Area $\left(\mathrm{m}^{2}\right)$ & $1.7(0.2)$ & $1.4-2.0$ \\
\hline \multicolumn{3}{|l|}{$\operatorname{Sex}(\%)$} \\
\hline Male & 40 & \\
\hline Female & 60 & \\
\hline ALT (IU/L) & $45.1(20.1)$ & $19.0-100$ \\
\hline AST (IU/L) & $38.9(19.4)$ & $10.0-83.0$ \\
\hline Alkaline Phosphatase (IU/L) & $234(108)$ & $52-467$ \\
\hline Total Bilirubin $(\mu \mathrm{mol} / \mathrm{L})$ & $0.6(0.3)$ & $0.2-1.6$ \\
\hline Serum Albumin $(\mathrm{g} / \mathrm{L})$ & $33.3(10.6)$ & $14.9-50.5$ \\
\hline Total Protein $(\mathrm{g} / \mathrm{L})$ & $59.8(11.6)$ & $38.8-79.3$ \\
\hline Creatinine Clearance $^{a}(\mathrm{~mL} / \mathrm{min})$ & $85.3(32.9)$ & $23.2-150.0$ \\
\hline Hemoglobin $(\mathrm{g} / \mathrm{dL})$ & $11.3(1.5)$ & $6.4-13.0$ \\
\hline Leukocyte Count $\times 10^{9} / \mathrm{L}$ & $7.7(4.2)$ & $0.4-17.8$ \\
\hline Neutrophil $\times 10^{9} / \mathrm{L}$ & $7.5(5.8)$ & $1.7-26.5$ \\
\hline Platelets $\times 10^{9} / \mathrm{L}$ & $261(139)$ & $115-716$ \\
\hline \multicolumn{3}{|l|}{ Liver metastases } \\
\hline No (\%) & 83.3 & \\
\hline Yes $(\%)$ & 16.7 & \\
\hline Peritoneal Carcinomatosis Index & 8.6 & $0.0-39.0$ \\
\hline \multicolumn{3}{|l|}{ Complete Cytoreduction } \\
\hline No $(\%)$ & 33.3 & \\
\hline Yes $(\%)$ & 66.7 & \\
\hline
\end{tabular}

Continuous variables are expressed as mean (standard deviation) and range, whereas categorical variables are expressed as percentage (\%) ${ }^{a}$ Creatinine Clearance was calculated using the Cockroft and Gault's formula and values higher than $150 \mathrm{~mL} / \mathrm{min}$ were truncated to $150 \mathrm{~mL} / \mathrm{min}$ resect all visible tumor lesions larger than $2.5 \mathrm{~mm}$. Anastomoses were carried out after the perfusion of the abdominal cavity was completed. The cytoreduction obtained by surgery was considered complete $\mathrm{CC} 0$ (no residual implants remained), incomplete $\mathrm{CC} 1$ (residual implants $<2.5 \mathrm{~mm}$ persisted), incomplete CC2 (residual implants $\geq 2.5 \mathrm{~mm}$, but $<2.5 \mathrm{~cm}$ persisted) or incomplete CC3 (residual implants $\geq 2.5 \mathrm{~cm}$ persisted) (34).

Hyperthermic Intraperitoneal Oxaliplatin. An open coliseum technique was used according to the procedure previously described (35). Four 36-Fr drains were connected to a continuous closed circuit, and two intraperitoneal thermal probes were placed in order to obtain a proper temperature feedback. Briefly, a Tenckhoff inflow catheter was placed centrally in the abdomen and four outflow catheters were inserted through separate stab incisions in the abdominal wall. Both the inflow and outflow catheters were connected to a perfusion pump and heat exchanger. The skin of the abdomen was attached to a retractor ring, and the abdominal cavity was covered with a plastic sheet with a small opening in the center allowing entrance for the surgeon's hands to stir the abdominal contents in order to deliver a more uniform drug distribution and heat to the intra-abdominal surfaces. The rollers of an extracorporeal circulation machine (Performer LRT, Rand) were set at a speed of $1,000 \mathrm{~mL} / \mathrm{min}$ to deliver the perfusate, $4 \%$ icodextrin solution. The circuit passed through a heat exchanger which raised the temperature to $48^{\circ} \mathrm{C}$. The perfusate temperature on the abdominal cavity fluctuated between $42^{\circ} \mathrm{C}$ and $43^{\circ} \mathrm{C}$. Once the temperature was achieved, oxaliplatin $360 \mathrm{mg} / \mathrm{m}^{2}$ was administered. The perfusate volume varied from patient to patient depending on the peritoneal surface area and, approximately, 2.5 to $6.0 \mathrm{~L}$ were employed. On average, the HIO mean duration was 40 min (range 30$60 \mathrm{~min}$ ). After the end of perfusion, the solution was evacuated. During the next five postoperative days, 5fluorouracil (5-FU) was administered at a dose of $15 \mathrm{mg} / \mathrm{kg}$ intraperitoneally in $1 \mathrm{~h}$ infusion through a 14-Fr catheter in order to potentiate the oxaliplatin cytotoxic effect (36).

Sample Collection and Bioanalytical Methods. Peritoneal fluid and venous blood samples were collected immediately after the oxaliplatin administration and then every $10 \mathrm{~min}$ until the end of the peritoneal perfusion. Additional venous blood samples were drawn at $0.25,0.5,1,1.5,2,2.5,3,4,6,8$, $12,16,20,24$, and $28 \mathrm{~h}$ after the end of the peritoneal perfusion. All samples were collected in S-monovette ${ }^{\circledR}$ tubes, centrifuged at 3,500 rpm for $10 \mathrm{~min}$ and were stored at $-80^{\circ} \mathrm{C}$ until analysis. Total platinum in peritoneal fluid and plasma was measured using a validated assay based on inductively coupled plasma atomic emission spectrometry. The lower limit of quantification was $0.5 \mathrm{mg} / \mathrm{L}$. Over the validated range of the assay $(0.5$ to $30 \mathrm{mg} / \mathrm{L}$ for plasma samples and 5 to $300 \mathrm{mg} / \mathrm{L}$ for peritoneal fluid samples), the mean intra- and inter-assay coefficients of variation were lower than $9.5 \%$ and $7.7 \%$, respectively. Blood samples for the determination of ANC were collected before the surgery and, afterwards, daily until patient completely recovered from the hematological toxicity. ANC were determined using an automated hematology analyzer (Beckman Coulter, Inc. AcT5diff AL, Fullerton, CA, USA). 
Pharmacokinetic and Pharmacodynamic Model Development

Software. Nonlinear mixed-effects modeling using the first-order conditional (FOCE) method implemented in NONMEM VI software package (ICON, Hanover, MD) (37) was used to develop the population PK/PD model and to conduct model-based simulations. Compilations were achieved using DIGITAL Visual Fortran Version 6.6C. Graphical and all other statistical analyses were performed using S-Plus 6.1 Professional Edition (Insightful, Seattle, WA, USA).

Pharmacokinetic and Pharmacodynamic Model. Oxaliplatin concentrations in peritoneal fluid evidenced a monoexponential decay, and consequently, oxaliplatin in the peritoneal fluid was assumed to be absorbed into plasma according to a linear process, characterized by the first-order absorption rate constant, $k_{\mathrm{a}}$ (Fig. 1). As oxaliplatin concentration in the peritoneal fluid were available, the absorption process was parameterized in terms of peritoneum to plasma clearance $\left(Q_{\mathrm{a}}\right)$ and volume of distribution in the peritoneum $\left(V_{\mathrm{a}}\right)$; thus $k_{\mathrm{a}}$ was calculated as a secondary parameter $\left(Q_{\mathrm{a}} / V_{\mathrm{a}}\right)$. Based on the graphical exploratory analysis, the oxaliplatin disposition in plasma was characterized by an open two-compartment model with linear elimination and nonspecific distribution to peripheral tissues (Fig. 1). This model was parameterized in terms of systemic clearance $(C l)$, intercompartmental flow $\left(Q_{2}\right)$, central volume of distribution $\left(V_{\mathrm{c}}\right)$, and peripheral volume of distribution $\left(V_{\mathrm{p}}\right)$. As the oxaliplatin absolute bioavailability $(F)$ after intraperitoneal administration is not known, $F$ was fixed to 1 ; therefore, the estimated model parameters were apparent. The corresponding differential equations for each compartment were:

$$
\begin{gathered}
\frac{d A}{d t}=-\frac{Q_{\mathrm{a}}}{V_{\mathrm{a}}} \cdot A=-k_{\mathrm{a}} \cdot A \\
\frac{d C}{d t}=\frac{Q_{\mathrm{a}}}{V_{\mathrm{a}}} \cdot A-\frac{Q_{2}}{V_{\mathrm{c}}} \cdot C-\frac{C l}{V_{\mathrm{c}}} \cdot C+\frac{Q_{2}}{V_{\mathrm{p}}} \cdot P \\
\frac{d P}{d t}=\frac{Q_{2}}{V_{\mathrm{c}}} \cdot C-\frac{Q_{2}}{V_{\mathrm{p}}} \cdot P
\end{gathered}
$$

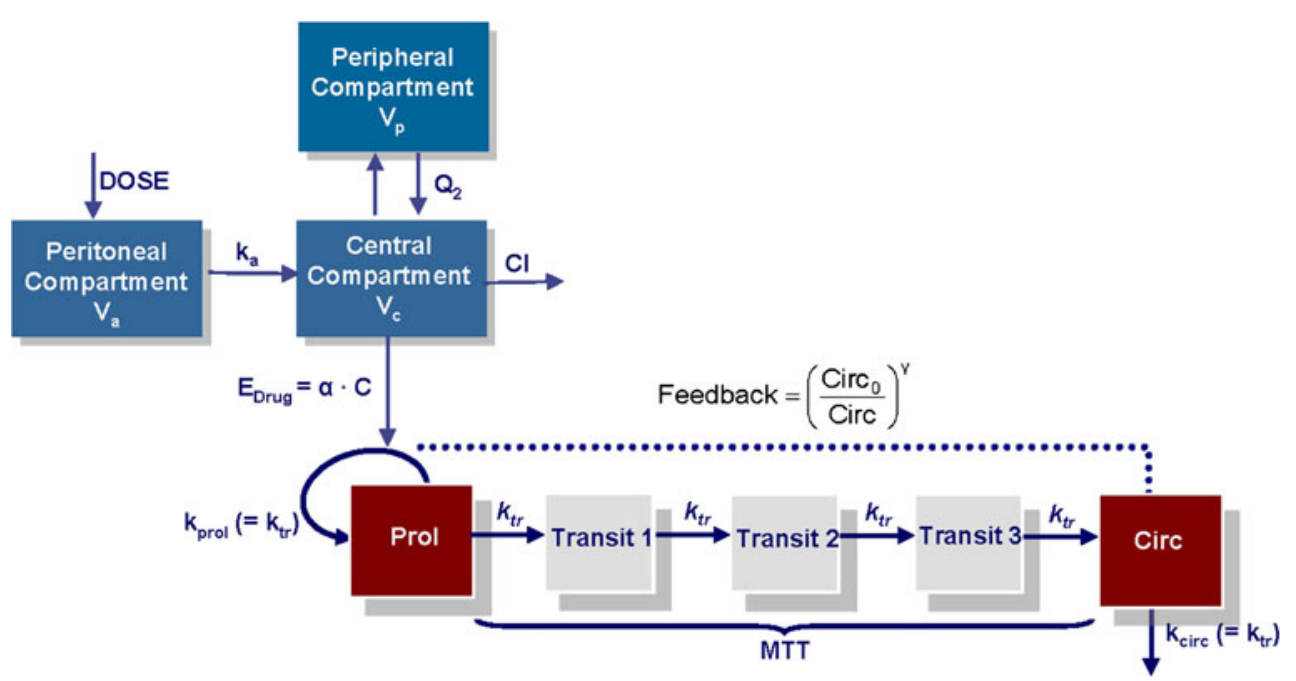

Fig. 1. Schematic of the semi-mechanistic population PK/PD model where $A, C$, and $P$ represent the oxaliplatin concentrations in peritoneal fluid, plasma, and peripheral compartment, respectively.

The semi-mechanistic model proposed by Friberg et al. (30) was used to describe the ANC time course as a function of oxaliplatin concentrations (Fig. 1). The backbone structure of the model consist in five compartments: one compartment represents the proliferative cells [Prol], such as stem cell and other progenitor cells; three transit compartments with maturating cells [Transit]; and one compartment of the circulating blood cells [Circ]. A maturation chain, with transit compartments and first-order rate constants $\left(k_{\mathrm{tr}}\right)$ accounts for the lag time between the oxaliplatin administration and the observed neutropenic effects in blood. The generation of new cells in [Prol] was dependent on the number of cells in that compartment, which is consistent with the mechanism of self-renewal or mitosis. The first-order proliferation rate constant, $k_{\text {prol }}$, determines the rate of cell division, together with the feedback mechanism from the circulating cells. The feedback loop was necessary to describe the rebound of ANC compared to the baseline values $\left(\operatorname{Circ}_{0}\right)$ and was incorporated into the model as $\left(\operatorname{Circ}_{0} / \operatorname{Circ}\right)^{\gamma}$ as previously suggested (35). The feedback function is governed by the $\gamma$ parameter, which reflects the increase in self-replication rate occurring when circulating cells are depleted. The differential equations describing the reference model were as follows:

$$
\begin{gathered}
\frac{d \text { Prol }}{d t}=k_{\text {Prol }} \cdot \text { Prol } \cdot\left(\frac{\text { Circ }_{0}}{\text { Circ }^{\gamma}}\right)^{\gamma} \cdot\left(1-E_{\text {Drug }}\right)-k_{\mathrm{tr}} \cdot \text { Prol } \\
\frac{d \text { Transit }_{1}}{d t}=k_{\mathrm{tr}} \cdot \text { Prol }-k_{\mathrm{tr}} \cdot \text { Transit }_{1} \\
\frac{d \text { Transit }_{2}}{d t}=k_{\mathrm{tr}} \cdot \text { Transit }_{1}-k_{\mathrm{tr}} \cdot \text { Transit }_{2} \\
\frac{d \text { Transit }_{3}}{d t}=k_{\mathrm{tr}} \cdot \text { Transit }_{2}-k_{\mathrm{tr}} \cdot \text { Transit }_{3} \\
\frac{d \text { Circ }_{\text {ir }}}{d t}=k_{\mathrm{tr}} \cdot \text { Transit }_{3}-k_{\mathrm{Circ}} \cdot \text { Circ }
\end{gathered}
$$


The oxaliplatin plasma concentrations were assumed to reduce the proliferation rate according to a linear function ( $\left.E_{\text {Drug }}\right)$ :

$$
E_{\text {Drug }}=\alpha \cdot C
$$

where $\alpha$ is the slope of the linear relationship between $E_{\text {Drug }}$ and $C$, and $C$ is derived based on the empirical Bayesian estimates of the individual pharmacokinetic parameter obtained from the oxaliplatin population pharmacokinetic model previously described.

In the transit compartments, it was assumed that the only loss of cells is into the next compartments; therefore, the random loss of precursor cells was assumed to be negligible. As the proliferative cells differentiate into more mature cell types, the concentration of cells is maintained by cell division. At steadystate, before administering oxaliplatin, $d \mathrm{Prol} / d t$ is equal to 0 and, therefore, $k_{\mathrm{Prol}}=k_{\mathrm{tr}}$. As the ANC data collected did not contain enough information to estimate independently $k_{\text {Circ }}$, it was fixed to the population mean half life of neutrophils previously determined, $0.07 \mathrm{~h}^{-1}(30)$. To improve the interpretability, the mean transit time (MTT) was estimated as follows:

$$
\mathrm{MTT}=\frac{n+1}{k_{\mathrm{tr}}}
$$

where $n$ is the number of transit compartments. MTT represents the time taken for the neutrophil to reach the circulation after leaving the proliferative compartment. Thus the structural model parameters to be estimated were the system-related parameters: $\operatorname{Circ}_{0}, M T T$, and $\gamma$, and the drug-related parameter, $\alpha$.

The effect of 5-FU on the inhibition of the proliferation rate and/or stimulation of the killing rate of the progenitor cells was assumed to be negligible because the low intrinsic neutropenic effects of 5-FU (38) and the relatively low doses administered, which lead to a negligible systemic exposure as evidence by the large proportion $(81.5 \%)$ of 5 -FU plasma concentration below the limit of quantification $(0.04 \mathrm{mg} / \mathrm{L})$.

Statistical Model. The interindividual (or between subjects) variability (IIV) in the PK/PD model parameters was assumed to follow the lognormal distribution and, consequently, an exponential error model was used. Residual variability in oxaliplatin peritoneal concentrations, oxaliplatin plasma concentrations, or ANC was evaluated using an additive error model after natural logarithmic transformation of the observations and model predictions. The magnitude of interindividual and residual variability was expressed approximately as a coefficient of variation.

Model Selection Criteria. The improvement of the fit obtained for each model was assessed in several ways. First, the resulting NONMEM-generated minimum value of the objective function (MVOF) was used to perform the likelihood ratio test. This test is based on the change in the MVOF ( $\triangle \mathrm{MVOF}$ ), which is equal (up to a constant) to minus twice the log-likelihood of the data and is asymptotically distributed like $\chi^{2}$ with the degrees of freedom equal to the number of parameters added to the model. $\Delta$ MVOFs of -10.83 or -12.12 were required to reach statistical significance at $p \leq 0.0010$ or $p \leq 0.0005$ for the inclusion or exclusion of one fixed effect in nested models, respectively. These stringent statistical criteria were used to avoid the inclusion of weak and clinically no relevant effects. In addition, the improvement in the fit was assessed by the reduction in the IIV and residual variability, the precision in parameter estimates, and the examination of diagnostic plots, and shrinkage (39).

Model Qualification. A nonparametric bootstrap was used as internal evaluation method to qualify the estimates of the PK/PD model parameters (40) using WINGS for NONMEM (N. Holford, Version 6.16, Auckland, New Zealand). The mean and the $95 \%$ confidence intervals of the parameter estimates from the bootstrap replicates were compared with the estimated parameters from the original dataset. In addition, a visual predictive check was performed on the time course of the 5th, 50th, and 95th percentile of the oxaliplatin peritoneal and plasma concentrations and the ANC (41).

Model-Based Simulations. Based on the PK/PD model developed, simulations were undertaken in order to explore the role of the initial oxaliplatin concentration in the peritoneum (and therefore the dose) and the duration of the $\mathrm{HIO}$ on the incidence of neutropenia grade 4 or grade 4 lasting at least 5 days. For a total of 12 oxaliplatin concentrations $(0,25,50,75,100,125,150,175,200,225,250,275$, and $300 \mathrm{mg} / \mathrm{L})$, the daily ANC was simulated for four different HIO durations (30, 40, 50, and $60 \mathrm{~min})$ and the incidence of neutropenia grade 4 or grade 4 lasting at least 5 days was computed. For each scenario, 1,000 virtual subjects were simulated.

\section{RESULTS}

Pharmacokinetics. A total of 140 and 338 oxaliplatin concentrations from peritoneum and plasma, respectively, were available for the PK analysis. The mean (SD) of the $C_{\max }$ in peritoneum and plasma was determined to be 82.30 (17.76) $\mathrm{mg} / \mathrm{L}$ at $6.36(7.13) \mathrm{min}$ after the start of HIO and 2.56 (0.90) $\mathrm{mg} / \mathrm{L}$ at 35.97 (8.20) min after the start of HIO, respectively. Moreover, the mean (SD) of the area under the curve concentration $v s$. time curve (AUC) in peritoneum and plasma was determined to be 1,150 (348) and 87.20 (123.20) $\mathrm{mg} \cdot \mathrm{h} / \mathrm{L}$, respectively.

The time course of plasma concentrations following HIO was best described by an open two-compartment disposition model with nonspecific distribution to a peripheral compartment, linear elimination from the central compartment, and first-order absorption from peritoneum to plasma. Figure 2 displays the goodness-of-fit plots for oxaliplatin peritoneal concentrations (upper panels) and oxaliplatin plasma concentrations (mid-panels), which showed a normal random scatter around the identity line and indicated the absence of significant bias. The final estimates of the pharmacokinetic parameters and the results of the non-parametric bootstrap analysis are presented in Table II. Except for $Q_{2}$, between subject variability was estimated for all of the PK parameters, with acceptable shrinkage $(<0.3)$. The population estimates of model parameters were very similar to the mean of the 684 bootstrap replicates that minimized successfully and were contained within the $95 \%$ confidence intervals obtained from the bootstrap analysis, suggesting an acceptable accuracy of the parameters estimates. 

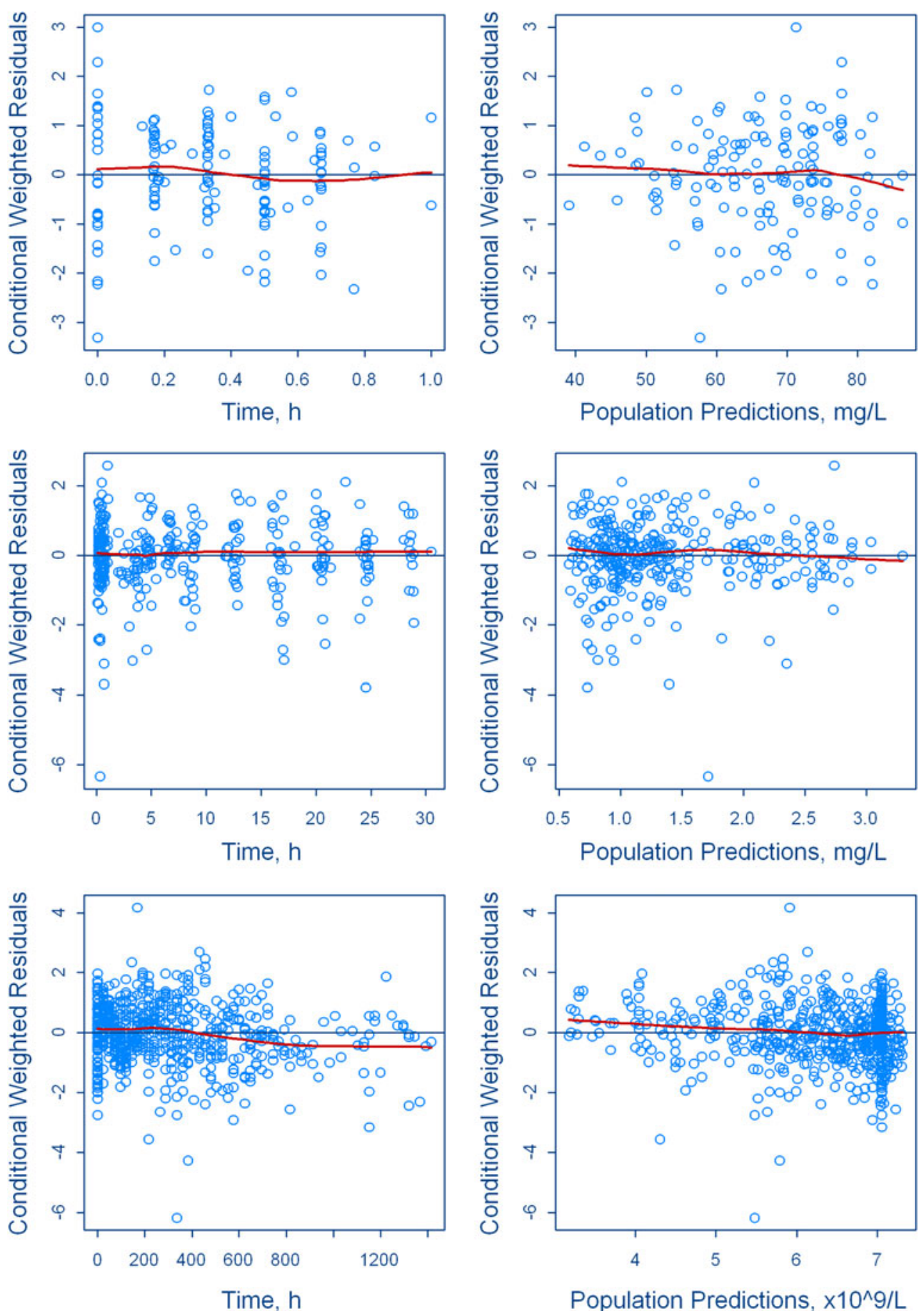

Fig. 2. Conditional weighted residuals $v s$ time and conditional weighted residuals $v s$ population predictions for peritoneal (upper panels) and plasma (mid panels) oxaliplatin concentrations, and absolute neutrophils counts (lower panels)

The precision of the fixed effects estimates was also good, with relative standard error (RSE) lower than $34 \%$, while the RSE for the random effect ranged from $30 \%$ to $70 \%$.

The results of the visual predictive check performed are depicted in Fig. 3. In this figure, the blue areas cover the $95 \%$ confidence interval of the $5^{\text {th }}, 50^{\text {th }}$, and $95^{\text {th }}$ percentiles of the model-based prediction for peritoneal or plasma concentrations and red solid lines represent the observed $5^{\text {th }}, 50^{\text {th }}$, and $95^{\text {th }}$ percentiles of the peritoneal or plasma concentrations. This figure evidence that the PK model developed is appropriate to describe the time course of peritoneal and plasma oxaliplatin concentrations and their associated variability observed in cancer patients with PC.

Pharmacodynamics. A total of 678 ANC values were available for the PK/PD analysis. The mean (SD) of the Circ 0 was determined to be $7.47 \cdot 10^{9} / \mathrm{L}\left(5.78 \cdot 10^{9} / \mathrm{L}\right)$ and remains relatively constant until the 4 to 6 days after drug 
Table II. Parameter Estimates and Bootstrap Analysis of the HIO Population Pharmacokinetic and Pharmacodynamic Model

\begin{tabular}{|c|c|c|c|}
\hline \multirow[b]{2}{*}{ Model parameters } & \multirow{2}{*}{$\frac{\text { Original dataset }}{\text { Estimate }^{a, b}}$} & \multicolumn{2}{|c|}{ Nonparametric bootstrap } \\
\hline & & Mean $^{a}$ & 95\% Confidence interval \\
\hline \multicolumn{4}{|l|}{ Pharmacokinetic model } \\
\hline$Q_{\mathrm{a}}(\mathrm{L} / \mathrm{h})$ & 2.70 & $2.80(17.3)$ & $1.91-3.86$ \\
\hline$V_{\mathrm{a}}(\mathrm{L})$ & 8.33 & $8.32(4.48)$ & $7.61-9.09$ \\
\hline $\mathrm{Cl} / \mathrm{F}(\mathrm{L} / \mathrm{h})$ & 1.61 & $1.65(33.4)$ & $0.71-2.85$ \\
\hline $\mathrm{Q}_{2} / \mathrm{F}(\mathrm{L} / \mathrm{h})$ & 77.0 & $79.4(18.6)$ & $56.7-112.0$ \\
\hline$V_{\mathrm{c}} / \mathrm{F}(\mathrm{L})$ & 19.2 & $20.0(25.2)$ & $11.4-30.8$ \\
\hline$V_{\mathrm{p}} / \mathrm{F}(\mathrm{L})$ & 72.8 & $75.3(17.2)$ & $50.1-103.9$ \\
\hline \multicolumn{4}{|c|}{ Interindividual variability $(\mathrm{CV} \%)$} \\
\hline$\omega_{\mathrm{Qa}}$ & 34.1 & $34.1(30.6)$ & $23.4-44.2$ \\
\hline$\omega_{\mathrm{Va}}$ & 17.7 & $17.4(38.3)$ & $10.1-23.5$ \\
\hline$\omega_{\mathrm{CL} / \mathrm{F}}$ & 85.6 & $93.4(69.6)$ & $38.2-159.0$ \\
\hline$\omega_{\mathrm{Vc} / \mathrm{F}}$ & 57.9 & $56.0(69.8)$ & $15.1-84.2$ \\
\hline$\omega_{\mathrm{Vp} / \mathrm{F}}$ & 23.5 & $24.4(69.0)$ & $6.70-39.3$ \\
\hline \multicolumn{4}{|l|}{ Residual variability (CV \%) } \\
\hline$\sigma_{1}$ (plasma) & 14.7 & $14.5(16.8)$ & $12.0-16.8$ \\
\hline$\sigma_{2}$ (peritoneum) & 16.5 & $16.3(27.9)$ & $12.6-21.0$ \\
\hline \multicolumn{4}{|l|}{ Pharmacodynamic model } \\
\hline \multicolumn{4}{|l|}{ System related parameters } \\
\hline $\operatorname{Circ}_{0}\left(\cdot 10^{9} / \mathrm{L}\right)$ & $7.05(6.88)$ & $7.07(6.75)$ & $6.26-8.09$ \\
\hline MTT (h) & 118 Fixed & - & - \\
\hline$\gamma$ & $0.135(11.9)$ & $0.133(25.4)$ & $0.016-0.209$ \\
\hline \multicolumn{4}{|l|}{ Drug-related parameter } \\
\hline$\alpha(\mathrm{L} / \mathrm{mg})$ & $0.182(36.5)$ & $0.181(62.8)$ & $0.048-0.505$ \\
\hline \multicolumn{4}{|c|}{ Interindividual variability $(\mathrm{CV} \%)$} \\
\hline$\omega_{\text {Circ0 }}$ & $42.3(27.5)$ & $40.6(29.6)$ & $27.3-52.1$ \\
\hline$\omega_{\mathrm{MTT}}$ & $32.8(53.2)$ & $31.2(155.9)$ & $5.6-75.6$ \\
\hline$\omega_{\alpha}$ & $141(31.7)$ & $145(160.7)$ & $73-250$ \\
\hline \multicolumn{4}{|l|}{ Residual variability (CV \%) } \\
\hline$\sigma$ & $49.7(17.6)$ & $49.1(19.4)$ & $41.1-58.7$ \\
\hline
\end{tabular}

${ }^{a}$ Results expressed as parameter (RSE relative standard error of parameter estimate, \%)

${ }^{b}$ The covariate step failed. Therefore, RSE of PK parameters are not provided

administration when ANC begin to decline and reached a nadir approximately 11 to 14 days after the start of the HIO. The median ANC nadir determined was $3.09 \cdot 10^{9} / \mathrm{L}$ and showed large variability with values ranging from 0.03 to $10.31 \cdot 10^{9} / \mathrm{L}$. The incidence of patients with neutrophil count less than $1.00 \cdot 10^{9} / \mathrm{L}$ and $0.5 \cdot 10^{9} / \mathrm{L}$, suggestive of neutropenia at least grades 3 and 4 , respectively, was $17 \%$ and $10 \%$, respectively. The infusion duration for the three patients that developed neutropenia grade 4 was 35,45 , and $45 \mathrm{~min}$, and the corresponding ANC nadir was $0.0023 \cdot 10^{9} / \mathrm{L}, 0.028 \cdot 10^{9} / \mathrm{L}$ and $0.13 \cdot 10^{9} / \mathrm{L}$.

The model proposed by Friberg et al. (30) fits the ANC profiles reasonably well. Figure 2 displays the goodness-of-fit plots for ANC (lower panels), which also showed a normal random scatter around the line of identity and indicate an absence of bias. The final estimates of the PD parameters and the results of the non-parametric bootstrap analysis are presented in Table II. Between subject variability was estimated for $\operatorname{Circ} 0$ and $\alpha$ with acceptable shrinkage $(0.077$ and 0.224 , respectively). The shrinkage for MTT was determined to be 0.391 . The population estimates of model parameters were very similar to the mean of the 964 bootstrap replicates that minimized successfully, and were contained within the $95 \%$ confidence intervals obtained from the bootstrap analysis, suggesting an acceptable accuracy of the PD parameters. The precision of the fixed effects estimates was acceptable, with RSE lower than $36.6 \%$. In addition, the precision for the random effect parameters was also adequate with RSE ranging from $27.5 \%$ to $53.2 \%$. The results of the visual predictive check performed are depicted in Fig. 3 and evidence that the model developed is appropriate to describe the time course of ANC in cancer patients following cytoreductive surgery and HIO.

The exploratory graphical analysis of the effect of age, sex, body weight, serum creatinine, albumin, serum ALT, serum AST, total bilirubin, hemoglobin, and hematocrit did not suggest any correlation between these covariates and PK/ PD parameters. Therefore, given the limited number of subjects included in the current analysis, a formal analysis of covariate effects on PK/PD parameters was not attempted.

Model-Based Simulations. Deterministic simulations (Fig. 4, upper panels) clearly show that the neutropenia is reversible, short-lasting, and non-cumulative. In addition, the initial HIO concentration (and the oxaliplatin dose) and the infusion duration are the main determinants of the severity and the duration of the neutropenia. As a consequence of the linear drug effect model, Fig. 4 (upper panels) shows that a proportional increase in the oxaliplatin exposure will lead to a proportional decrease in the ANC nadir. In addition, extending the duration of the HIO administration, for a given initial 

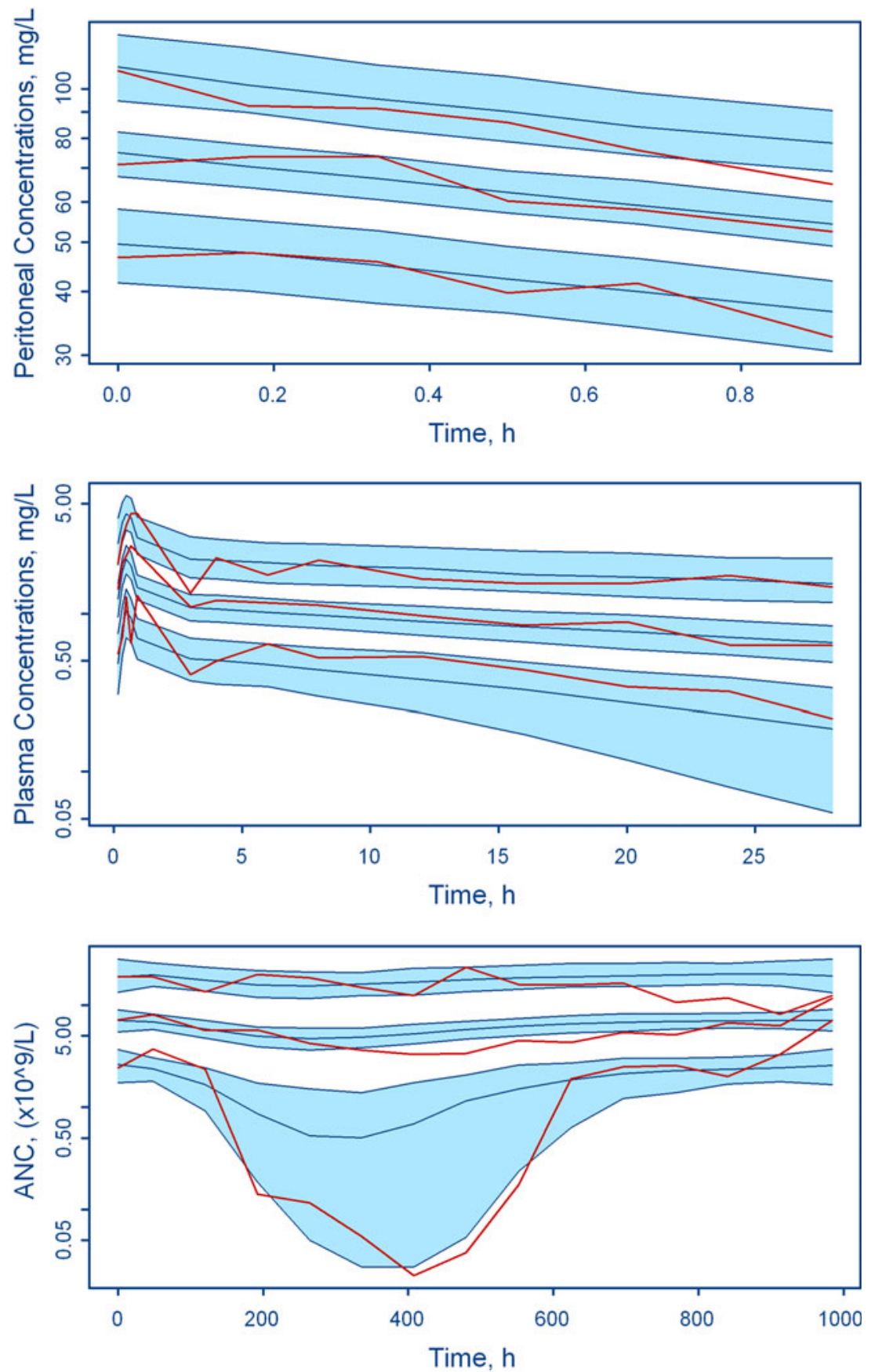

Fig. 3. Time course of the $5^{\text {th }}, 50^{\text {th }}$, and $95^{\text {th }}$ percentiles of the peritoneal (upper panel) and plasma (mid panel) oxaliplatin concentration and absolute neutrophils counts (lower panel) and their associated model-based prediction of the $95 \%$ confidence interval

oxaliplatin concentration in the peritoneum, will increase the severity and duration of the neutropenia as it is directly related to the oxaliplatin exposure in peritoneum.

The relationship between initial HIO concentration and the incidence of severe neutropenia is also displayed in Fig. 4 (lower panels) as a function of the infusion duration. In this figure, the incidence of neutropenia grade 4 appears linearly related to the initial $\mathrm{HIO}$ concentration and the slope of that linear relationship also depends of the infusion duration. Actually, a 60 -min infusion of oxaliplatin starting at peritoneal concentration of $65 \mathrm{mg} / \mathrm{L}$ leads to a $20 \%$ incidence of neutropenia Grade 4. However, a 30-min infusion starting at the same oxaliplatin concentration leads to a $12 \%$ incidence of neutropenia Grade 4. Thus, shorter HIO reduce the incidence of severe neutropenia, while the initial oxaliplatin dose administered to achieve an initial $65 \mathrm{mg} / \mathrm{L}$ concentration remains the same in both cases.

According to Fig. 4 (lower left panel), a 20\% incidence of neutropenia Grade 4 is also expected following a 30-min infusion starting at peritoneal oxaliplatin concentration of $105 \mathrm{mg} / \mathrm{L}$. Interestingly, the area under the peritoneal oxaliplatin concentration versus time curve (AUC) following a 60 -min infusion starting at $65 \mathrm{mg} / \mathrm{L}$ concentration is the same than the AUC following a 30-min infusion starting at 

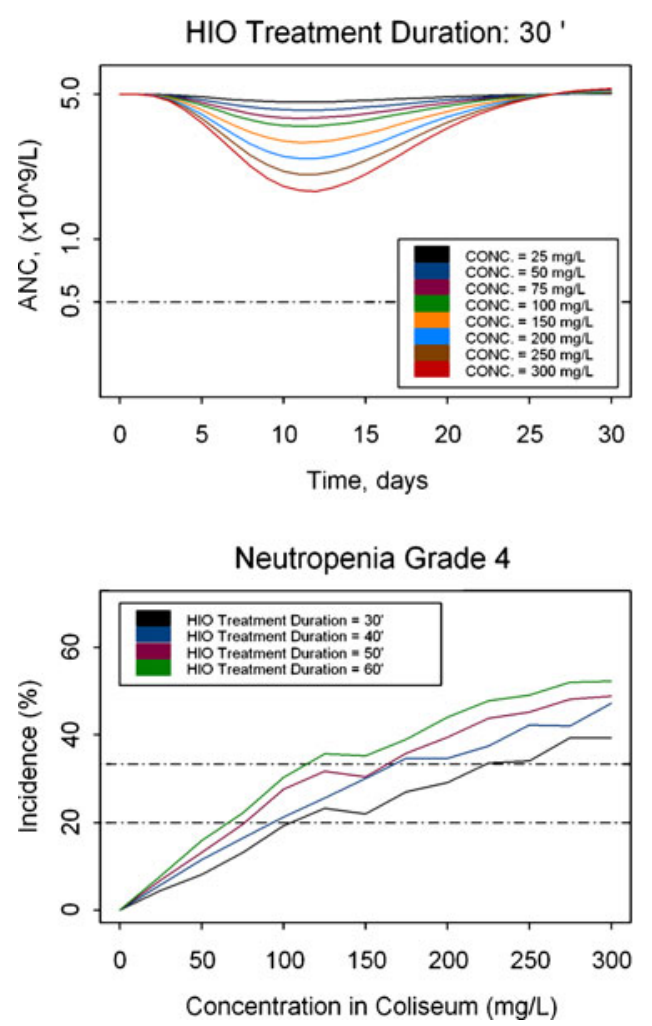

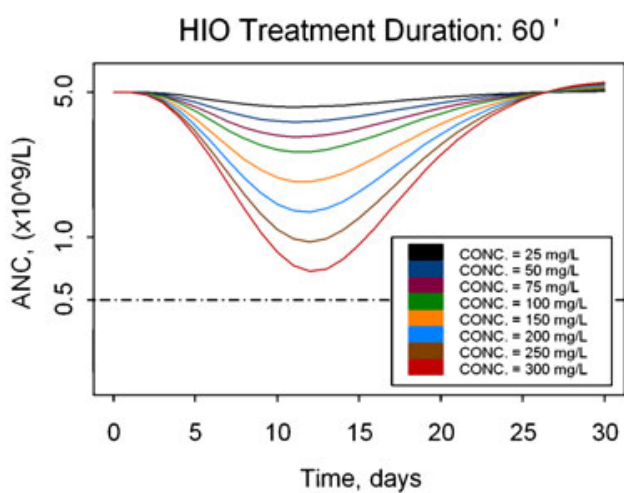

Neutropenia Grade 4 during 5 consecutive days

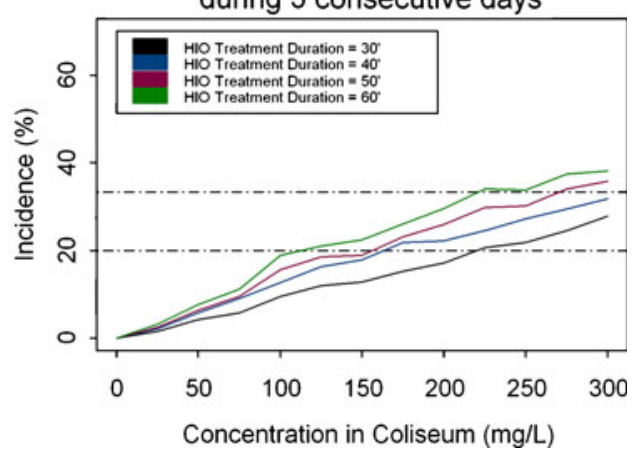

Fig. 4. Effect of initial oxaliplatin concentration in peritoneum and HIO treatment duration on the time course of neutrophil counts (upper panels) and on the incidence of neutropenia grade 4 and grade 4 lasting at least 5 days (lower panels)

oxaliplatin concentration of $105 \mathrm{mg} / \mathrm{L}$. Consequently, these two dosing regimens leads to an incidence of neutropenia grade 4 , for which primary prophylaxis with granulocytecolony stimulating factors is recommended. Furthermore, a 60 -min infusion starting at $120 \mathrm{mg} / \mathrm{L}$ concentration is associated with a $33 \%$ incidence of neutropenia grade 4 , which determine the maximum tolerated exposure. Similarly, HIO exposure of 120 and $225 \mathrm{mg} \mathrm{h} / \mathrm{L}$ are associated with a $20 \%$ and $33 \%$ incidence of neutropenia grade 4 lasting more than 5 days Fig. 4 (lower right panel).

\section{DISCUSSION}

In this study, the oxaliplatin pharmacokinetics in peritoneum and plasma has been characterized in cancer patients with PC treated with cytoreductive surgery followed by HIO. Regarding the oxaliplatin plasma disposition, the typical volume of the central compartment and the alpha half-life $\left(t_{1 / 2 \alpha}\right)$ were estimated to be $19.2 \mathrm{~L}$ and $0.14 \mathrm{~h}$, respectively, and were similar to the PK parameters previously reported by Ferron et al. (27) and Massari et al. (42) The beta half-life $\left(t_{1 /}\right.$ ${ }_{2 \beta}$ ) determined in the current study, $40 \mathrm{~h}$, was similar to that observed by Massari et al. $(37.5 \mathrm{~h})$ after 2-h intravenous infusion of oxaliplatin $130 \mathrm{mg} / \mathrm{m}^{2}$ but was longer than the $t_{1 / 2 \beta}$ reported by Ferron et al. $(12.9 \mathrm{~h})$. A possible explanation of these differences is the limited sampling period used to characterize the oxaliplatin pharmacokinetics in Ferron et al. study, as compared to the others studies. It becomes very difficult to accurately estimate oxaliplatin $t_{1 / 2 \beta}$ based on plasma samples collected only up to $8 \mathrm{~h}$ after the start of HIO administration. Sampling schedules including plasma concentration collected beyond $24 \mathrm{~h}$ should provide a more accurate estimation of the $t_{1 / 2 \beta}$ for total oxaliplatin.

The peritoneum to plasma ratio of oxaliplatin $C_{\max }, 32.1$, was similar to a previously reported value for ultrafiltrate platinum concentrations $(24,28)$. However, the apparent oxaliplatin peritoneal half-life $\left(t_{1 / 2 a}=\operatorname{Ln}(2) / k_{\mathrm{a}}\right)$, equivalent to the oxaliplatin absorption half-life from peritoneum to plasma, $2.2 \mathrm{~h}$, was considerably higher than the values reported previously (0.5-0.7 h) (25-27), probably because different carrier solution has been used in the current study. While all the previous pharmacokinetic studies of $\mathrm{HIO}$ were performed using isotonic $5 \%$ dextrose as carrier solution, this study reports, for the first time, the HIO pharmacokinetics in plasma and peritoneum using isotonic $4 \%$ icodextrin as carrier solution. Icodextrin is a macromolecule that, theoretically, should reduce oxaliplatin clearance from the peritoneal cavity and, consequently, the $t_{1 / 2 a}$ should be longer. On the other hand, the ratio estimated of the AUCs in peritoneum and plasma was 13.19 , which is in line with the values previously reported (28) for ultrafiltrate platinum using isotonic $5 \%$ dextrose as carrier solution. Probably, other factors, including differences in the surgical procedures, the extracorporeal circulation machines, the oxaliplatin absolute bioavailability and the analyte and bioanalytical method, could contribute to explain the differences in the absorption half-life from peritoneum to plasma across the studies conducted with dextrose at $5 \%$ vs icodextrin at $4 \%$. Therefore, further studies comparing oxaliplatin absorption with both carrier solutions are necessary to quantify the icodextrin effect on oxaliplatin absorption. 
In this study, the relationship between oxaliplatin pharmacokinetics and the time course of ANC in patients with $\mathrm{PC}$ receiving $\mathrm{HIO}$ after cytoreductive surgery was also investigated by applying a semi-mechanistic population PK/ PD model previously developed (30). The relationship between oxaliplatin plasma concentrations and drug effect was described by a linear function and the slope of the linear drug effect was estimated to be $0.182 \mathrm{~L} / \mathrm{mg}$. It appears that oxaliplatin neutropenic potency is about $28 \%$ higher than the estimated neutropenic potency for carboplatin in monotherapy, after correcting for the differences in the free fraction between the two drugs (43). The $5^{\text {th }}, 50^{\text {th }}$, and $95^{\text {th }}$ percentiles of the oxaliplatin peak inhibition of the proliferative rate of precursor cells into the Prol were determined to be 13.13, 44.23 , and 564.33, respectively. With respect to the system related parameters, the estimated of $\operatorname{Circ}_{0}$ was consistent with ANC normal values. The MTT could not be estimated correctly with the available data and, therefore, was fixed to $118 \mathrm{~h}$ as previously reported in the literature $(30,44-48)$. The estimated $\gamma$ value, 0.135 , was also similar to those obtained previously for other anticancer drugs, such as irinotecan $(0.132)$ or topotecan $(0.120)(30,49)$. Interindividual and residual variabilities were moderate to large, consistent with that observed for other drugs (30).

Model-based simulations revealed that HIO induced neutropenia is reversible, short-lasting, and largely dependent on the intensity of the dose administered (or concentration in the peritoneum) and the duration of the HIO treatment. Figure 4 (upper panels) shows that increasing the dose (or the initial concentration in the peritoneum) and/or extending the $\mathrm{HIO}$ duration leads to a greater fluctuation in ANC values and consequently increases the likelihood of severe neutropenia. Simulations also indicated that it is possible to reduce the degree of neutropenia by employing treatment regimens with shorter HIO duration, while the overall amount of dose administered (and the initial oxaliplatin concentration in the peritoneum) remains the same. Quantitatively, simulations suggest that the maximum tolerated HIO exposure is $120 \mathrm{mg} \cdot \mathrm{h} / \mathrm{L}$. However, primary prophylaxis with granulocyte-colony stimulating factors should be considered if $\mathrm{HIO}$ exposure is higher than $65 \mathrm{mg} \cdot \mathrm{h} / \mathrm{L}$ in order to prevent severe neutropenia as recommended by the guidelines (50).

In summary, a semi-mechanistic pharmacokinetic and pharmacodynamic model has been developed to account for the effect of oxaliplatin on myelosupression. This model has been successfully applied for the first time to describe the time course of ANC in cancer patients with diagnosis of PC treated with $\mathrm{HIO}$, using $4 \%$ icodextrin as carrier solutions. Model-based simulations suggest that targeting HIO exposure not higher than $120 \mathrm{mg} \cdot \mathrm{h} / \mathrm{L}$ is safe and, however, at exposure higher than $65 \mathrm{mg} \cdot \mathrm{h} / \mathrm{L}$ the primary prophylaxis with granulocyte-colony stimulating factors support is recommended. The model developed is useful to optimize the design of future clinical studies.

\section{ACKNOWLEDGMENTS}

The authors would like to thank the patients, medical, nursing, and laboratory staff of the USP Hospital San Jaime who participated in the present study. The authors indicated no potential conflicts of interest.

\section{REFERENCES}

1. Koga A, Watanabe K, Fukuyama T, et al. Diagnosis and operative indications for polypoid lesions of the gallbladder. Ann Surg. 1988;123:26-9.

2. Fujimoto S, Shrestha RD, Kokubun M, et al. Positive results of combined therapy of surgery and intraperitoneal hyperthermic perfusion for far-advanced gastric cancer. Ann Surg. 1990;212: $592-6$.

3. Fujimura T, Yonemura Y, Muraoka K, et al. Continuous hyperthermic peritoneal perfusion for the prevention of peritoneal recurrence of gastric cancer: randomized controlled study. World J Surg. 1994;18:150-5.

4. Alexander HR, Hanna N, Pingpank JF. Clinical results of cytoreduction and HIPEC for malignant peritoneal mesothelioma. Cancer Treat Res. 2007;134:343-55.

5. Witkamp AJ, de Bree E, Kaag MM, et al. Extensive cytoreductive surgery followed by intra-operative hyperthermic intraperitoneal chemotherapy with mitomycin-C in patients with peritoneal carcinomatosis of colorectal origin. Eur J Cancer. 2001;37:979-84.

6. Lentz SS, Miller BE, Kucera GL, Levine EA. Intraperitoneal hyperthermic chemotherapy using carboplatin: a phase I analysis in ovarian carcinoma. Gynecol Oncol. 2007;106:207-10.

7. Sugarbaker PH. Intraperitoneal chemotherapy and cytoreductive surgery for the prevention and treatment of peritoneal carcinomatosis and sarcomatosis. Semin Surg Oncol. 1998;14:254-61.

8. Los G, Mutsaers PH, van der Vijgh WJ, et al. Direct diffusion of cisplatinum in intraperitoneal rat tumors after intraperitoneal chemotherapy: a comparison with systemic chemotherapy. Cancer Res. 1989;48:3380-4.

9. Elias D, Ouellet JF. Intraperitoneal chemohyperthermia. Rationale, technique, indications and results. Surg Oncol Clin N Am. 2001; 10:915-33.

10. Verwaal V, van Ruth S, de Bree E, et al. Randomized trial of cytoreduction and hyperthermic intraperitoneal chemotherapy versus systemic chemotherapy and palliative surgery in patients with peritoneal carcinomatosis of colorectal cancer. J Clin Oncol. 2003;21:3737-43.

11. Verwaal V, van Ruth S, Witkamp A, et al. Long term survival of peritoneal carcinomatosis of colorectal origin. Ann Surg Oncol. 2005;12:65-71.

12. Dedrick RL, Flessner MF. Pharmacokinetic problems in peritoneal drug administration: tissue penetration and surface exposure. J Natl Cancer Inst. 1997:89:480-7.

13. Cho HK, Lush RM, Bartlett DL, et al. Pharmacokinetics of cisplatin administered by continuous hyperthermic peritoneal perfusion to patients with peritoneal carcinomatosis. J Clin Pharmacol. 1999:39:1-8.

14. Fujimoto S, Takahashi M, Mutou T, et al. Survival time and prevention of side effects of intraperitoneal hyperthermic perfusion with mitomycin $\mathrm{C}$ combined with surgery for patients with advanced gastric cancer. Cancer Treat Res. 1996;81:169-76.

15. Steller MA, Egorin MJ, Trimble EL, et al. A pilot phase I trial of continuous hyperthermic peritoneal perfusion with high-dose carboplatin as primary treatment of patients with small-volume residual ovarian cancer. Cancer Chemother Pharmacol. 1999;43:106-14.

16. de Bree E, Rosing H, Filis D, et al. Cytoreductive surgery and intraoperative hyperthermic intraperitoneal chemotherapy with paclitaxel: a clinical and pharmacokinetic study. Ann Surg Oncol. 2008;15:1183-92.

17. Elias D, Goere D, Blot F, et al. Optimization of hyperthermic intraperitoneal chemotherapy with oxaliplatin plus irinotecan at 43 degrees $\mathrm{C}$ after compete cytoreductive surgery: mortality and morbidity in 106 consecutive patients. Ann Surg Oncol. 2007;14:1818-24.

18. Elias D, Pocard M, Goere D. HIPEC with oxaliplatin in the treatment of peritoneal carcinomatosis of colorectal origin. Cancer Treat Res. 2007;134:303-18.

19. Elias D, Lefevre JH, Chevalier J, et al. Complete cytoreductive surgery plus intraperitoneal chemohyperthermia with oxaliplatin for peritoneal carcinomatosis of colorectal origin. J Clin Oncol. 2009;27:681-5. 
20. Urano M, Ling CC. Thermal enhancement of melphalan and oxaliplatin cytotoxicity in vitro. Int J Hyperthermia. 2002;18:307-15.

21. Atallah D, Marsaud V, Radanyi C, et al. Thermal enhancement of oxaliplatin-induced inhibition of cell proliferation and cell cycle progression in human carcinoma cell lines. Int J Hyperthermia. 2004;20:405-19.

22. Elias D, Bonnay M, Puizillou JM, et al. Heated intraoperative intraperitoneal oxaliplatin after complete resection of peritoneal carcinomatosis: pharmacokinetics and tissue distribution. Ann Oncol. 2002;13:267-72.

23. Culy CR, Clemett D, Wiseman LR. Oxaliplatin. A review of its pharmacological properties and clinical efficacy in metastasic colorectal cancer and its potential in other malignancies. Drugs. 2000;60:895-924.

24. Elias D, Sideris L. Pharmacokinetics of heated introperative intreperitoneal oxaliplatin after complete resection of peritoneal carcinomatosis. Surg Oncol Clin N Am. 2003;12:755-69.

25. Elias D, Raynard B, Bonnay M, Pocard M. Heated intraoperative intraperitoneal oxaliplatin alone and in combination with intraperitoneal irinotecan: Pharmacologic studies. Eur J Surg Oncol. 2006;32:607-13.

26. Stewart JH, Shen P, Russell G, et al. A phase I trial of oxaliplatin for intraperitoneal hyperthermic chemoperfusion for the treatment of peritoneal surface dissemination from colorectal and appendiceal cancers. Ann Surg Oncol. 2008;15:2137-45.

27. Ferron G, Dattez S, Gladieff L, et al. Pharmacokinetics of heated intraperitoneal oxaliplatin. Cancer Chemother Pharmacol. 2008;62:679-83.

28. Mahteme H, Wallin I, Glimelius B, et al. Systemic exposure of the parent drug oxaliplatin during hyperthermic intraperitoneasl perfusion. Eur J Clin Pharmacol. 2008;64:907-11.

29. Mohamed F, Sugarbaker PH. Carrier solutions for intraperitoneal chemotherapy. Surg Oncol Clin N Am. 2003;12:813-24.

30. Friberg LE, Henningsson A, Maas $\mathrm{H}$, et al. Model of chemotherapy-induced myelosuppression with parameter consistency across drugs. J Clin Oncol. 2002;20:4713-21.

31. Bretcha-Boix P, Farré-Alegre J, Sureda M, et al. Cytoreductive surgery and perioperative intraperitoneal chemotherapy in patients with peritoneal carcinomatosis of colonic origin: outcomes after 7 years' experience of a new centre for peritoneal surface malignancies. Clin Transl Oncol. 2010;12:437-42.

32. Sugarbaker PH. Intraperitoneal chemotherapy and cytoreductive surgery for the prevention and treatment of peritoneal carcinomatosis and sarcomatosis. Sem Surg Oncol. 1998;14:254-61.

33. Sugarbaker PH. Peritonectomy procedures. Ann Surg. 1995;221:29-42.

34. Jacquet P, Sugarbaker PH. Clinical research methodologies in diagnosis and staging of patients with peritoneal carcinomatosis. Cancer Treat Res. 1996;82:359-74.

35. Sugarbaker PH, Jacquet P, Stephens AD, et al. Comparison of covered versus closed technique for heated intraoperative intraperitoneal chemotherapy for peritoneal carcinomatosis from gastrointestinal cancer. In: Abe $\mathrm{O}$, Inokuchi K, Takasaki K, editors. Monduzzi Editore. Bologna: XXX World Congress of the International College of Surgeons; 1996. p. 389-93.

36. Giachetti S, Perpoint B, Zidani R, et al. Phase III multicenter randomized trial of oxaliplatin added to chronomoduled fluorouracil-leucovorin as first line treatment of metastasic colorectal cancer. J Clin Oncol. 2000;18:136-47.

37. Beal SL, Sheiner LB, Boeckman AJ (ed) NONMEM users guides. ICON Development Solutions, Ellicott City, 1989-2006.

38. Sandström M, Lindman H, Nygren P, et al. Population analysis of the pharmacokinetics and the haematological toxicity of the fluorouracil-epirubicin-cyclophosphamide regimen in breast cancer patients. Cancer Chemother Pharmacol. 2006;58:143-56.

39. Karlsson MO, Savic RM. Diagnosing model diagnostics. Clin Pharmacol Ther. 2007;82:17-20.

40. Efron B, Tibshirani R. An introduction to the bootstrap. New York: Chapman \& Hall/CRC; 1993.

41. Yano Y, Beal SL, Sheiner LB. Evaluating pharmacokinetic/ pharmacodynamic models using the posterior predictive check. J Pharmacokinet Pharmacodyn. 2001;28:171-92.

42. Massari C, Brienza S, Rotarski M, et al. Pharmacokinetics of oxaliplatin in patients with normal versus impaired renal function. Cancer Chemother Pharmacol. 2000;45:157-64.

43. Schmitt A, Gladieff L, Laffont CM, et al. Factors for hematopoietic toxicity of carboplatin: refining the targeting of carboplatin systemic exposure. J Clin Oncol. 2010;28:4568-74.

44. Léger F, Loos WJ, Bugat R, et al. Mechanism-based models for topotecan-induced neutropenia. Clin Pharmacol Ther. 2004;76:567-78.

45. Latz JE, Karlsson MO, Rusthoven JJ, et al. A semimechanisticphysiologic population pharmacokinetic/pharmacodynamic model for neutropenia following pemetrexed therapy. Cancer Chemother Pharmacol. 2006;57:412-26.

46. Sandström M, Lindman H, Nygren $\mathrm{P}$, et al. Model describing the relationship between pharmacokinetics and hematologic toxicity of the epirubicin-docetaxel regimen in breast cancer patients. J Clin Oncol. 2005;23:413-21.

47. Trocóniz IF, Garrido MJ, Segura C, et al. Phase I dose-finding study and a pharmacokinetic/pharmacodynamic analysis of the neutropenic response of intravenous diflomotecan in patients with advanced malignant tumors. Cancer Chemother Pharmacol. 2006;57:727-35.

48. Kathman SJ, Williams DH, Hodge JP, et al. A Bayesian population PK-PD model of ispinesib-induced myelosuppression. Clin Pharmacol Ther. 2007;81:88-94.

49. Hing J, Perez Ruixo JJ, Stuyckens K, et al. Mechanism-based pharmacokinetic/pharmacodynamic meta-analysis of trabectedin (ET-743, Yondelis) induced neutropenia. Clin Pharmacol Ther. 2008;83:130-43.

50. Aapro MS, Cameron DA, Pettengell R, et al. EORTC guidelines for the use of granulocyte-colony stimulating factor to reduce the incidence of chemotherapy-induced febrile neutropenia in adult patients with lymphomas and solid tumors. Eur J Cancer. 2006; $42: 2433-53$. 